ZASTOSOWANIA MATEMATYKI APPLICATIONES MATHEMATICAE XVIII, 4 (1985). p.517-523

V. SCHMIDT (Freiberg)

\title{
ON INFINITE-SERVER QUEUES WITH CYCLICALLY DEPENDENT SERVICE TIMES
}

1. Introduction. It is well known that in the infinite-server queue $M / G I / \infty$ with Poisson arrival moments and with identically distributed independent service times, which are also independent of the arrival moments, the stationary distribution of the number of customers in the system is Poisson with parameter depending on the arrival intensity and on the mean service time only, but not on the form of the service time distribution. The question, in which we are interested, is for what class of stationary sequences of (possibly dependent) service times this insensitivity property is preserved.

In the following we propose a way to consider an infinite-server queue with Poisson arrivals and with service times, which are cyclically dependent in some sense stated below. For this queue we show that the stationary distribution of the number of customers in the system is also Poisson with parameter being equal to the arrival intensity multiplied by the mean service time. Moreover, if the service times satisfy some mixing condition this stationary distribution can be approximated by the corresponding timedependent distributions under the condition that a busy period starts at time zero.

2. The model. Assume that the arrival moments $\left\{T_{n}\right\}$ form a homogeneous Poisson process with intensity $\lambda(0<\lambda<\infty)$. Furthermore, let be given a strictly stationary sequence $\left\{S_{n}\right\}, S_{n}=\left(S_{n}^{1}, S_{n}^{2}, \ldots\right)$, of infinitely dimensional random vectors consisting of independent identically distributed non-negative components $\left(0<E S_{n}^{k}<\infty\right)$. Besides the assumption that for every fixed $n$ the random variables $S_{n}^{1}, S_{n}^{2}, \ldots$ are independent, we additionally assume that the sequences $\left\{\boldsymbol{S}_{n}\right\}$ and $\left\{T_{n}\right\}$ are independent. 
Thereby, $S_{n}^{1}$ is the service time of the customer who first arrives during the $n$th busy period, $S_{n}^{2}$ the service time of the second arriving customer during the $n$th busy period and so on. Thus, the service times of the customers served during the same busy period are assumed to be independent. On the other hand, concerning service times being realized on different busy periods no independence assumptions are made.

Assume that $T_{1}=0$ and that at time zero the first busy period starts. Thus, the customer arriving at time zero receives the service time $S_{1}^{1}$.

Because the behaviour of the considered queueing system on a given busy period is the same as that of the $M / G I / \infty$ queue with completely independent service times, from a result of Shanbhag [4] we get the finiteness of the mean busy period Ex:

$$
\mathrm{E} \varkappa=\lambda^{-1}(\exp (\lambda / \mu)-1)<\infty,
$$

where $\mu^{-1}=E S_{n}^{k}$. This means that the stationary sequence of cycles

$$
\begin{array}{r}
\left\{\left(T_{1}, S_{1}^{1}\right],\left[T_{2}, S_{1}^{2}\right], \ldots,\left[T_{N_{1}}, S_{1}^{N_{1}}\right]\right),\left(\left[T_{N_{1}+1}, S_{2}^{1}\right],\left[T_{N_{1}+2}, S_{2}^{2}\right], \ldots\right. \\
\left.\left.\ldots,\left[T_{N_{1}+N_{2}}, S_{2}^{N_{2}}\right]\right), \ldots\right\},
\end{array}
$$

where $N_{n}$ denotes the number of customers being served during the $n$th busy period, generates a Palm point process distribution, see [1] and [2].

Namely, it induces the conditional Palm distribution $P_{e}$ of the marked point process $\left\{\left[T_{i},\left(S_{i}, \Gamma_{i}\right)\right]\right\}$ of the arrival moments $T_{i}$ marked by the corresponding (actually realized) service time $S_{i}$ and by the indicator $\Gamma_{i}$ under the condition that the customer arriving at time zero finds no other customers in the system, i.e. he initiates a busy period. Thereby, $S_{i}=S_{n+1}^{k}$ if $i=N_{1}+\ldots+N_{n}+k \leqslant N_{1}+\ldots+N_{n+1}$ and

$$
\Gamma_{i}= \begin{cases}1 & \text { if } i=N_{1}+\ldots+N_{n}+1 \text { for some } n, \\ 0 & \text { otherwise }\end{cases}
$$

In other words, $P_{e}$ is the Palm distribution of $\left\{\left[T_{i},\left(S_{i}, \Gamma_{i}\right)\right]\right\}$ with respect to the mark set $R_{+} \times\{1\}$.

Furthermore, using a result of Neveu [3] (see also Theorem 1.4.5 in [1]), from $P_{e}$ we get the unconditional Palm distribution $P_{0}$ of $\left\{\left[T_{i},\left(S_{i}, \Gamma_{i}\right)\right]\right\}$ :

$$
P_{0}(\cdot)=\frac{1}{\mathrm{E} N_{1}} \int \sum_{j=1}^{N_{1}} I_{(\cdot)}\left(\theta_{j} \varphi\right) P_{e}(d \varphi),
$$

where $\varphi=\left\{\left[t_{i},\left(s_{i}, \gamma_{i}\right)\right]\right\}$ and $\theta_{j} \varphi=\left\{\left[t_{i}-t_{j},\left(s_{i}, \gamma_{i}\right)\right]\right\} ; I_{X}$ denotes the indicator of the set $X$. Thus, $P_{0}$ is the Palm distribution of $\left\{\left[T_{i},\left(S_{i}, \Gamma_{i}\right)\right]\right\}$ with respect to the set $R_{+} \times\{0,1\}$ of all possible marks. Note that $P_{0}$ is $\theta_{j}$-invariant for every $j$. 


\section{The number of customers in the system.}

THEOREM 1. We have

$$
P_{0}(\varphi: \xi(\varphi)=k)=\frac{(\lambda / \mu)^{k}}{k !} \exp (-\lambda / \mu), \quad k=0,1,2, \ldots,
$$

where

$$
\xi(\varphi)=\sum_{i} f\left(t_{i}(\varphi), s_{i}(\varphi)\right) \quad \text { and } \quad f(t, s)= \begin{cases}1 & \text { if } t<0, s>-t \\ 0 & \text { otherwise }\end{cases}
$$

To prove Theorem 1 we need two lemmas.

Lemma 1. For every $t, t^{\prime}<0\left(t \neq t^{\prime}\right)$ we have

$$
P_{0}\left(s_{i}>-t, s_{j}>-t^{\prime} \mid t_{i}=t, t_{j}=t^{\prime}\right)=(1-B(-t))\left(1-B\left(-t^{\prime}\right)\right), \quad i, j \leqslant 0,
$$

where $B(x)$ is the distribution function of the service times $S_{n}^{k} ; B(x)$ $=P\left(S_{n}^{k} \leqslant x\right)$.

Proof. This statement results from the above made independence assumptions and from the form (1) of the probability measure $P_{0}$. Without loss of generality we assume that $t<t^{\prime}$, i.e. $i<j$. Then, from (1) we get

$$
\begin{aligned}
& P_{0}\left(s_{i}>-t, s_{j}>-t^{\prime}, t_{i} \in\{d t\}, t_{j} \in\left\{d t^{\prime}\right\}\right) \\
&= \frac{1}{\mathrm{E} N_{1}} \sum_{k=1}^{\infty} P_{e}\left(\varphi: N_{1}(\varphi) \geqslant k, s_{i}\left(\theta_{k} \varphi\right)>-t, s_{j}\left(\theta_{k} \varphi\right)>-t^{\prime},\right. \\
&\left.t_{i}\left(\theta_{k} \varphi\right) \in\{d t\}, t_{j}\left(\theta_{k} \varphi\right) \in\left\{d t^{\prime}\right\}\right) \\
&= \frac{1}{\mathrm{E} N_{1}} \sum_{k=1}^{\infty} P_{e}\left(\varphi: N_{1}(\varphi) \geqslant k, s_{i+k}(\varphi)>-t, s_{j+k}(\varphi)>-t^{\prime},\right. \\
&= \frac{1}{\mathrm{E} N_{1}} \sum_{k=-i+1}^{\infty} P_{e}\left(\varphi: N_{1}(\varphi) \geqslant k, s_{i+k}(\varphi)>-t, s_{j+k}(\varphi)>-t_{k+1},\right. \\
&=\left.\frac{1}{\mathrm{E} N_{1}} \sum_{k=-i+1}^{\infty} P_{e}\left(\varphi: N_{1}(\varphi) \geqslant k d t\right\}, t_{j+k}(\varphi)-t_{k+1}(\varphi) \in\left\{d t^{\prime}\right\}\right) \\
&=(1-B(-t))\left(1-B\left(-t^{\prime}\right)\right) \frac{1}{\left.\operatorname{EN}_{1+k}(\varphi)-t_{k+1}(\varphi) \in\{d t\}, t_{k+1}(\varphi) \in\{d t\}, t_{j+k}(\varphi)-t_{k+1}(\varphi) \in\left\{d t^{\prime}\right\}\right)} P_{e}\left(\varphi: N_{1}(\varphi) \geqslant k+i, s_{j+k}(\varphi)>-t^{\prime},\right. \\
&\left.t_{i+k}(\varphi)-t_{k+1}(\varphi) \in\{d t\}, t_{j+k}(\varphi)-t_{k+1}(\varphi) \in\left\{d t^{\prime}\right\}\right)
\end{aligned}
$$




$$
\begin{aligned}
& =(1-B(t))\left(1-B\left(-t^{\prime}\right)\right) \frac{1}{E N_{1}} \sum_{k=1}^{\infty} P_{e}\left(\varphi: N_{1}(\varphi) \geqslant k,\right. \\
& \left.\qquad t_{k}(\varphi)-t_{-i+1+k}(\varphi) \in\{d t\}, t_{j-i+k}(\varphi)-t_{-i+1+k}(\varphi) \in\left\{d t^{\prime}\right\}\right) \\
& =(1-B(-t))\left(1-B\left(-t^{\prime}\right)\right) P_{0}\left(t_{0}-t_{-i+1} \in\{d t\}, t_{j-i}-t_{-i+1} \in\left\{d t^{\prime}\right\}\right) \\
& =(1-B(-t))\left(1-B\left(-t^{\prime}\right)\right) P_{0}\left(t_{i} \in\{d t\}, t_{j} \in\left\{d t^{\prime}\right\}\right) .
\end{aligned}
$$

LEMMA 2. With respect to the probability measure $P_{0}$, the random variables $\left\{t_{i}\right\}$ also form a Poisson process with intensity $\lambda$.

Proof. From (1) we get

$$
\begin{aligned}
P_{0}\left(t_{n+1}\right. & \left.-t_{n}<x_{n}, \ldots, t_{2}-t_{1}<x_{1}\right) \\
& =\frac{1}{\mathrm{E} N_{1}} \sum_{j=1}^{\infty} P_{e}\left(N_{1} \geqslant j, t_{n+1+j}-t_{n+j}<x_{n}, \ldots, t_{2+j}-t_{1+j}<x_{1}\right) \\
& =\frac{1}{\mathrm{E} N_{1}} \sum_{j=1}^{\infty} P_{e}\left(N_{1} \geqslant j\right) P_{e}\left(t_{n+1+j}-t_{n+j}<x_{n}, \ldots, t_{2+j}-t_{1+j}<x_{1}\right) \\
& =P_{e}\left(t_{n+1}-t_{n}<x_{n}, \ldots, t_{2}-t_{1}<x_{1}\right)
\end{aligned}
$$

for every $n=1,2, \ldots$ and for every $x_{1}, \ldots, x_{n} \geqslant 0$.

Proof of Theorem 1. The formula (2) is equivalent with the statement that with respect to the probability measure $P_{0}$ the factorial moments of $\xi$ have the following form:

(3) $\mathrm{E}_{P_{0}}[\xi(0)(\xi(0)-1)(\xi(0)-2) \ldots(\xi(0)-(k-1))]=(\lambda / \mu)^{k}, \quad k=1,2, \ldots$

For brevity, we provide the proof of (3) for $k=2$ only. For $k \neq 2$ similar arguments can be used. We have

$$
\begin{aligned}
\mathrm{E}_{P_{0}}[\xi(0)(\xi(0)-1)] & =\int\left\{\sum_{i} f\left(t_{i}(\varphi), s_{i}(\varphi)\right)\left[\sum_{j} f\left(t_{j}(\varphi), s_{j}(\varphi)\right)-1\right]\right\} P_{0}(d \varphi) \\
& =\int \sum_{i \neq j} f\left(t_{i}(\varphi), s_{i}(\varphi)\right) f\left(t_{j}(\varphi), s_{j}(\varphi)\right) P_{0}(d \varphi) \\
& =\int_{R^{2} \times R_{+}^{2}} f(t, s) f\left(t^{\prime}, s^{\prime}\right) \alpha_{P_{0}}^{(2)}\left(d\left(t, s, . t^{\prime}, s^{\prime}\right)\right),
\end{aligned}
$$

where $\alpha_{P_{0}}^{(2)}$ is the second factorial moment measure of $P_{0}$;

$$
\begin{aligned}
\alpha_{P_{0}}^{(2)}\left(X_{1} \times L_{1} \times X_{2}\right. & \left.\times L_{2}\right) \\
& =\int \sum_{i \neq j} I_{X_{1} \times L_{1}}\left(t_{i}(\varphi), s_{i}(\varphi)\right) I_{X_{2} \times L_{2}}\left(t_{j}(\varphi), s_{j}(\varphi)\right) P_{0}(d \varphi) .
\end{aligned}
$$


Thus, from Lemma 1 we get

$$
\begin{aligned}
\mathrm{E}_{P_{0}} & {[\xi(0)(\xi(0)-1)] } \\
= & \sum_{i \neq j} \int_{R^{2}} \int_{R_{+}^{2}} f(t, s) f\left(t^{\prime}, s^{\prime}\right) P_{0}\left(s_{i} \in\{d s\}, s_{j} \in\left\{d s^{\prime}\right\} \mid t_{i}=t, t_{j}=t^{\prime}\right) \times \\
& \times P_{0}\left(t_{i} \in\{d t\}, t_{j} \in\left\{d t^{\prime}\right\}\right) \\
= & \sum_{i \neq j} \int_{R_{-}^{2}} P_{0}\left(s_{i}>-t, s_{j}>-t^{\prime} \mid t_{i}=t, t_{j}=t^{\prime}\right) P_{0}\left(t_{i} \in\{d t\}, t_{j} \in\left\{d t^{\prime}\right\}\right) \\
= & \sum_{i \neq j} \int_{R_{-}^{2}}(1-B(-t))\left(1-B\left(-t^{\prime}\right)\right) P_{0}\left(t_{i} \in\{d t\}, t_{j} \in\left\{d t^{\prime}\right\}\right) \\
= & \int_{R_{-}^{2}}(1-B(-t))\left(1-B\left(-t^{\prime}\right)\right) \alpha^{(2)}\left(d\left(t, t^{\prime}\right)\right),
\end{aligned}
$$

where $\alpha^{(2)}\left(X_{1} \times X_{2}\right)=\alpha_{P_{0}}^{(2)}\left(X_{1} \times R_{+} \times X_{2} \times R_{+}\right)$. This gives (3) taking into consideration that from I.emma 2 we have $\alpha^{(2)}\left(d\left(t, t^{\prime}\right)\right)=\lambda^{2} d t d t^{\prime}$ for $t, t^{\prime}<0$.

Note that in completion to (1) it is possible to express the distribution $P_{e}$ by $P_{0}$, too.

\section{THEOREM 2.}

$$
P_{e}(\cdot)=P_{0}(\cdot \mid \xi=0)
$$

Proof. From (1) we get

$$
\begin{aligned}
P_{0}(\{\cdot\} \cap\{\xi=0\}) \\
=\frac{1}{\mathrm{E} N_{1}} \int \sum_{j=1}^{N_{1}} I_{\{\cdot\} \cap\left\{\psi: \sum_{i \leqslant 0} I_{\left\{-t_{i}<s_{i}\right\}}(\psi)=0\right\}}\left(\theta^{j} \varphi\right) P_{e}(d \varphi) \\
=\frac{1}{\mathrm{E} N_{1}} \int I_{\{\cdot\} \cap\left\{\psi: \sum_{i \leqslant 0} I_{\left\{-t_{i}<s_{i}\right\}}(\psi)=0\right\}}\left(\theta^{N_{1}(\varphi)} \varphi\right) P_{e}(d \varphi) \\
=\frac{1}{\mathrm{E} N_{1}} \int I_{\{\cdot\} \cap\left\{\psi: \sum_{i \leqslant 0} I_{\left\{-t_{i}<s_{i}\right\}}(\psi)=0\right\}}(\varphi) P_{e}(d \varphi) \\
=\frac{1}{\mathrm{E} N_{1}} P_{e}\left(\{\cdot\} \cap\left\{\psi: \sum_{i \leqslant 0} I_{\left\{-t_{i}<s_{i}\right\}}(\psi)=0\right\}\right)=\frac{1}{\mathrm{E} N_{1}} P_{e}(\cdot)
\end{aligned}
$$

and, furthermore, $P_{0}(\xi=0)=\left(E N_{1}\right)^{-1}$.

4. Ergodic theorem. Now, we turn towards the question under what conditions we have the convergence

$$
\lim _{n \rightarrow \infty} P_{e}\left(\varphi: \xi_{n}(\varphi)=k\right)=P_{0}(\varphi: \xi(\varphi)=k)
$$


where

$$
\xi_{n}(\varphi)=\sum_{i=1}^{n-1} I_{\left\{t_{n}-t_{i}<s_{i}\right\}}(\varphi)
$$

Therefore, $\xi_{n}$ is the number of customers which are in the system immediately before the arrival moment of the $n$th customer, under the condition that at time $T_{1}=0$ a busy period starts.

The strictly stationary sequence $\left\{S_{n}\right\}$ of service time vectors is called strong mixing if for every $n$

$$
\sup _{Z_{\in \in \mathscr{F}^{n}}{ }_{-\infty}, Z^{\prime} \in \mathscr{F}_{n+m}^{\infty}}\left|P\left(Z \cap Z^{\prime}\right)-P(Z) P\left(Z^{\prime}\right)\right| \underset{m \rightarrow \infty}{\longrightarrow} 0,
$$

where $\mathscr{F}_{a}^{b}$ denotes the $\sigma$-algebra generated by the random vectors $\left\{\boldsymbol{S}_{i} ; i=a, \ldots, b\right\}$.

THEOREM 3. The mixing condition (5) is sufficient for the validity of (4).

Proof. First we note that without any additional condition we have

$$
\lim _{n \rightarrow \infty} P_{0}\left(\xi_{n}=k\right)=P_{0}(\xi=k)
$$

for every $k=0,1, \ldots$ (see Theorem 2.3 .1 in [1]). Thus, the statement is proved if we show that

$$
\lim _{n \rightarrow \infty} P_{e}\left(\xi_{n}=k\right)=\lim _{n \rightarrow \infty} P_{0}\left(\xi_{n}=k\right)
$$

for every $k=0,1, \ldots$ Using formula (1), the probability $P_{0}\left(\xi_{n}=k\right)$ can be written as follows:

$$
\begin{gathered}
P_{0}\left(\varphi: \xi_{n}(\varphi)=k\right)=P_{0}\left(\varphi: \sum_{i=1}^{n-1} I_{\left\{t_{n}-t_{i}<s_{i}\right\}}(\varphi)=k\right) \\
=\frac{1}{\operatorname{E} N_{1}} \sum_{r=1}^{\infty} P_{e}\left(N_{1}=r\right) \sum_{j=1}^{r} P_{e}\left(\left\{\varphi: \sum_{i=1}^{n-1} I_{\left\{t_{n+j}-t_{i+j}<s_{i+j}\right\}}(\varphi)=k\right\} \mid\left\{\varphi: N_{1}(\varphi)=r\right\}\right) .
\end{gathered}
$$

By the dominated convergence theorem $\left(\mathrm{E} N_{1}<\infty\right)$, instead of the limit

$$
\lim _{n \rightarrow \infty} \sum_{r=1}^{\infty} P_{e}\left(N_{1}=r\right) \sum_{j=1}^{r} P_{e}\left(\left\{\varphi: \sum_{i=1}^{n-1} I_{\left\{t_{n+j}-t_{i+j}<s_{i+j}\right\}}(\varphi)=k\right\} \mid\left\{\varphi: N_{1}(\varphi)=r\right\}\right) \text {, }
$$

for every pair $(r, j)$ the limit

$$
\lim _{n \rightarrow \infty} P_{e}\left(\left\{\varphi: \sum_{i=1}^{n-1} I_{\left\{i_{i+j} ;-t_{i+j}<s_{i+j}\right\}}(\varphi)=k\right\} \mid\left\{\varphi: N_{1}(\varphi)=r\right\}\right)
$$


can be considered. Note that for every $\varepsilon>0$ and for every $m>0$ we have

$$
\begin{aligned}
& \mid P_{e}\left(\left\{\varphi: \sum_{i=1}^{n-1} I_{\left\{t_{n+j}-t_{i+j}<s_{i+j}\right\}}(\varphi)=k\right\} \mid\left\{\varphi: N_{1}(\varphi)=r\right\}\right)- \\
& \quad-P_{e}\left(\left\{\varphi: \sum_{i=N_{1}+\ldots+N_{m}}^{n-1} I_{\left\{t_{n+j}-t_{i+j}<s_{i+j}\right.}(\varphi)=k\right\} \mid\left\{\varphi: N_{1}(\varphi)=r\right\}\right) \mid<\varepsilon
\end{aligned}
$$

for all sufficiently large $n$. Moreover, taking into consideration the independence of the sequences $\left\{T_{n}\right\}$ and $\left\{S_{n}\right\}$, from (5) we get

$$
\begin{aligned}
\mid P_{e}\left(\left\{\varphi: \sum_{i=N_{1}+\ldots+N_{m}}^{n-1} I_{\left\{t_{n+j}-t_{i+j}<s_{i+j}\right\}}(\varphi)=k\right\} \mid\left\{\varphi: N_{1}(\varphi)=r\right\}\right)- \\
\quad-P_{e}\left(\varphi: \sum_{i=N_{1}+\ldots+N_{m}}^{n-1} I_{\left\{t_{n+j}-t_{i+j}<s_{i+j}\right\}}(\varphi)=k\right) \mid<\varepsilon
\end{aligned}
$$

for all sufficiently large $m$.

\section{References}

[1] P. Franken, D. König, U. Arndt and V. Schmidt, Queues and point processes, Akademie-Verlag, Berlin 1981 and J. Wiley \& Sons, New York 1982.

[2] D. König, K. Matthes and K. Nawrotzki, Verallgemeinerungen der Erlangschen und Engsetschen Formeln (Eine Methode in der Bedienungstheorie), Akademie-Verlag, Berlin 1967.

[3] J. Neveu, Sur les mesures de Palm de deux processus ponctuels stationnaires, Z. Wahrsch. Verw. Gebiete 34 (1976), p. 199-203.

[4] D. N. Shanbhag, On infinite server queues with batch arrivals, J. Appl. Probab. 3 (1966), p. 274-279.

DEPARTMENT OF MATHEMATICS

MINING ACADEMY OF FREIBERG

DDR-9200 FREIBERG

Received on 3. 2.1983

2 - Zastosowania Mat. 18/4 